\title{
Qualitätsbestimmung von RFID-Komponenten auf der Basis von allgemein anerkannten Normen und Richtlinien - Vereinfachung von Ausschreibungen
}

\author{
Frank Gillert, Hardy Zissel
}

Der Einsatz von RFID Systemen in Bibliotheken wird längst nicht mehr nur in der Gruppe der sogenannten »Early Adopters« diskutiert, sondern ist auf der Tagesordnung, wenn es um die Modernisierung von Bibliotheken geht. Seit kurzem werden Richtlinienarbeiten zum Einsatz von RFID-Systemen auf den Weg gebracht. Ziel ist es, ausgehend von einer ausschreibungsrelevanten Leistungsmetrik auch praktikable Abnahme- und Testverfahren abzuleiten, die gemäß dem Stand der Technik branchenweite Anerkennung finden. Um dieses Ziel zu erreichen, ist es zum einen von Bedeutung, die Anwender eng einzubinden, zum anderen alle weltweit bedeutenden Herstellerunternehmen zu involvieren. Ebenso sollte die Erarbeitung ganzheitlich erfolgen, indem Methoden des Qualitätsmanagements berücksichtigt werden.

Nicht zuletzt müssen Beschreibungen des Stands der Technik formal seitens offizieller Standardisierungsinstitutionen möglichst international publizierbar sein. Es wird ein Stufenmodell vorgestellt, das eine sofort verwertbare, deutsch-englische VDI-Richtlinie enthält, die direkt zur Unterstützung von Ausschreibungen genutzt werden kann und darauf aufbauend über die geeigneten Wege in den Standardisierungsprozess ISO integriert werden kann.

\section{Einführung}

Die logistischen Abläufe innerhalb einer Bibliothek sind vielfältig, zeitaufwendig und im Sinne des Lean Managements existieren Prozessteile, die der »Verschwendung « zu zuordnen sind. Derartige Prozessteile sind insb. zu vermeiden, da sie von hochqualifizierten Bibliothekaren zu Ungunsten der eigentlichen Aufgaben im Rahmen des Wissensmanagements vorgenommen werden. Um den Anforderungen an eine moderne Bibliothekslandschaft gerecht zu werden, die sich durch freien Zugang durch den Nutzer zu den Medien zu allen Zeiten (24/7) sowie flexible Ausleihe und Rückgabeprozesse auszeichnet, halten mehr und mehr innovative Lösungen aus den Bereichen Informations- und Kommunikationstechnologien Einzug in die Infrastruktur der Bibliotheken. Schwerpunkte sind automatische Identifikation, mobile Datenerfassungsgeräte sowie Ortungs-, Lokalisierungs- und Visualisierungstechnologien. Im Folgenden wird die RFID Technologie im Hinblick auf Bibliotheksprozesse näher betrachtet. 


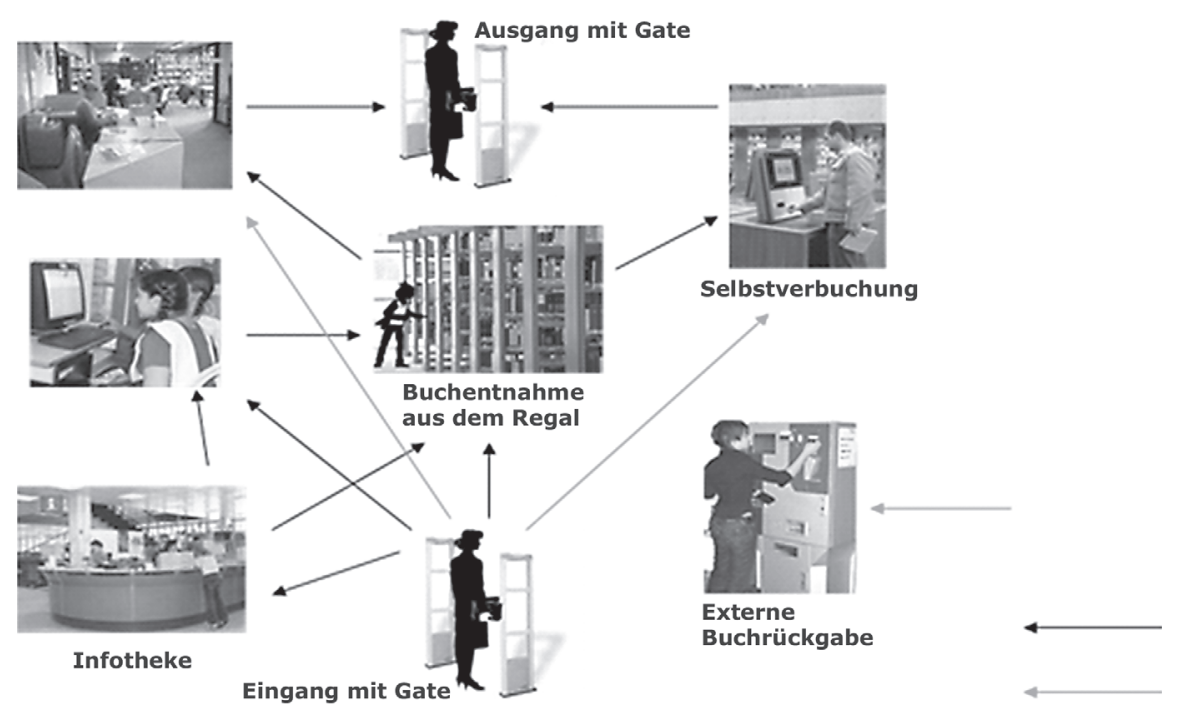

Abb. 1: Intralogistik-Abläufe in einer Bibliothek aus Sicht des Benutzers

Um diese Abläufe zu unterstützen, werden unterschiedliche RFID-Teilsysteme benötigt. Eine Einteilung von RFID-Systemen innerhalb von Bibliotheken lässt sich dabei folgendermaßen untergliedern:

- RFID Schleusensysteme

- RFID Selbstverbuchungsplätze

- RFID Rückgabeautomaten und Sortierstrecken

- RFID Personalarbeitsplätze

- RFID Handlesegeräte zur Inventarisierung

- RFID Konvertierungsplätze

- Transponder/Etiketten

Eine Unterscheidung der einzelnen Systeme ist wichtig, da je nach Einsatzort und Aufgabe andere Faktoren auf diese einwirken. Das können zum einen Umweltfaktoren sein, die sich unmittelbar aus der Umgebung herleiten lassen. Es können aber auch Einflussfaktoren sein, die sich aus der Benutzung des Gerätes erschließen. Um eine objektive Beurteilung der Leistungsfähigkeit zu erreichen, müssen daher die einzelnen Systeme getrennt betrachtet werden. Dies erfolgt im Rahmen dieses Konzeptes in der Analyse der funktionalen Parameter, getrennt für jedes Teilsystem.

Grundsätzlich kann gesagt werden, dass die Funktionsweise aller Teilsysteme ähnlich ist, da die Kommunikation über die Luftschnittstelle mit den Transpondern standardisiert erfolgt. Dabei besitzt jedes System ein Lesegerät, welches in Kombination mit einer Antenne mit einem Transponder kommuniziert. Das Lesegerät besitzt eine Schnittstelle zu einem IT-System auf dem eine Middleware läuft. Über diese Middleware werden die Kommunikationsprotokolle des Lesegerätes umgewandelt und für die weitere Verarbeitung beispielsweise mit dem Library Managementsystem (LMS) aufbereitet. Ab da erfolgt die Kommunikation über die für Bibliotheken typischen Schnittstellen und Protokolle. 


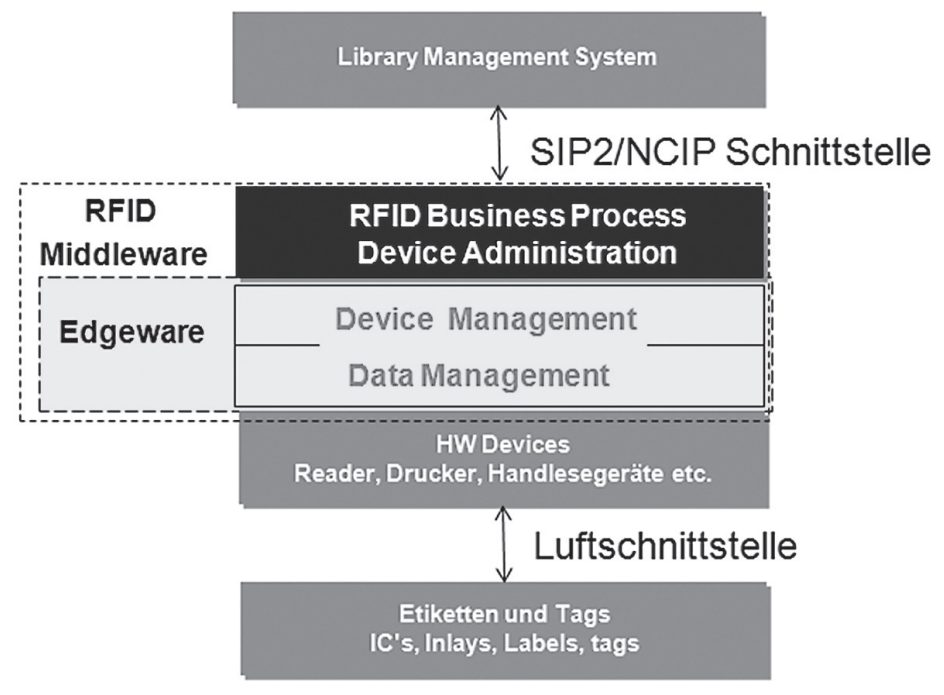

Abb. 2: Schema Teil-RFID-System in einer Bibliothek [in Anlehnung an Gill2007]

Bezüglich der Anforderungen an RFID-Systeme in Bibliotheken konnten verschieden Einflussgrößen auf die einzelnen Teilsysteme identifiziert werden. Je nach Verwendungszweck eines Teilsystems ergeben sich unterschiedlichste Bewertungskennzahlen und Einflussgrößen. In den Analysen der Teilsysteme dieses Konzeptes werden diese Parameter spezifiziert und darauf aufbauend die zu entwickelnden Prüfverfahren hergeleitet.

\section{Definitionen Qualitätsmanagement}

Unter Qualität versteht man die Gesamtheit der Merkmale und Merkmalswerte eines Produktes oder einer Dienstleistung bezüglich ihrer Eignung, festgelegte und vorausgesetzte Erfordernisse zu erfüllen. [vgl. ISO 8402]

Voraussetzung für das zu konzipierende Qualitätsmanagement für die RFID-Nutzung in Bibliotheken sind Qualitätsstandards mit klar definierten Anforderungen an RFIDBibliothekssysteme.

Ausgangspunkt sind die Anforderungen bisheriger Ausschreibungen hinsichtlich Funktionalität, Sicherheit, Zuverlässigkeit, Wartbarkeit, Anpassbarkeit usw. Damit die Qualität von RFID-Systemen bereits im Vorfeld und auch während der unterschiedlichen Projektphasen sichergestellt werden kann, ist eine ganzheitliche Qualitätssicherung notwendig. Diese umfasst alle geplanten und systematischen Tätigkeiten, die innerhalb eines Qualitätsmanagementsystems verwirklicht sind und die solche Qualitätsanforderungen erfüllen.

Das Qualitätsmanagement selbst umfasst dabei sowohl die Arbeitsmittel zur Erfüllung dieser Anforderungen, als auch neben der Qualitätssicherung alle Formen der Qualitätspolitik, Qualitätsplanung und Qualitätsverbesserung. 
Die Abgrenzungen der einzelnen Begriffe werden in der Qualitätsmanagement-Normenreihe DIN EN ISO 9000 ff. sowie in den zu den Normen dazugehörigen Qualitätsmanagement-Elementen aufgezeigt. Die Werkzeuge und Methoden des Qualitätsmanagements lassen sich je nach Zielsetzung in Methoden und Werkzeuge zur Qualitätsplanung, zur Produktrealisierung, zur Qualitätsauswertung und zur Qualitätsverbesserung systematisieren. Diese sind jedoch komplex. In Anlehnung an die Normenreihe DIN EN ISO 9000 ff. werden daher praxistaugliche Maßnahmen vorgeschlagen, welche die Fehlererkennung und Fehlervermeidung im Bezug auf RFID-Systeme in Bibliotheken in vereinfachterem Umfang realisiert.

Für die Implementierung neuartiger RFID-Bibliothekssysteme werden in den unterschiedlichen Projektstadien unterschiedliche Qualitätssicherungsmaßnahmen (Abb. 3) benötigt. Bereits zu Beginn einer Ausschreibung (Stufe 1) sind präventive Qualitätsmanagement-Prozesse notwendig, die es ermöglichen, bereits in dieser frühen Phase wesentliche Anforderungen zu validieren. Somit lassen sich Fehler vermeiden, die später möglicherweise mit hohen Kosten verbunden sind.

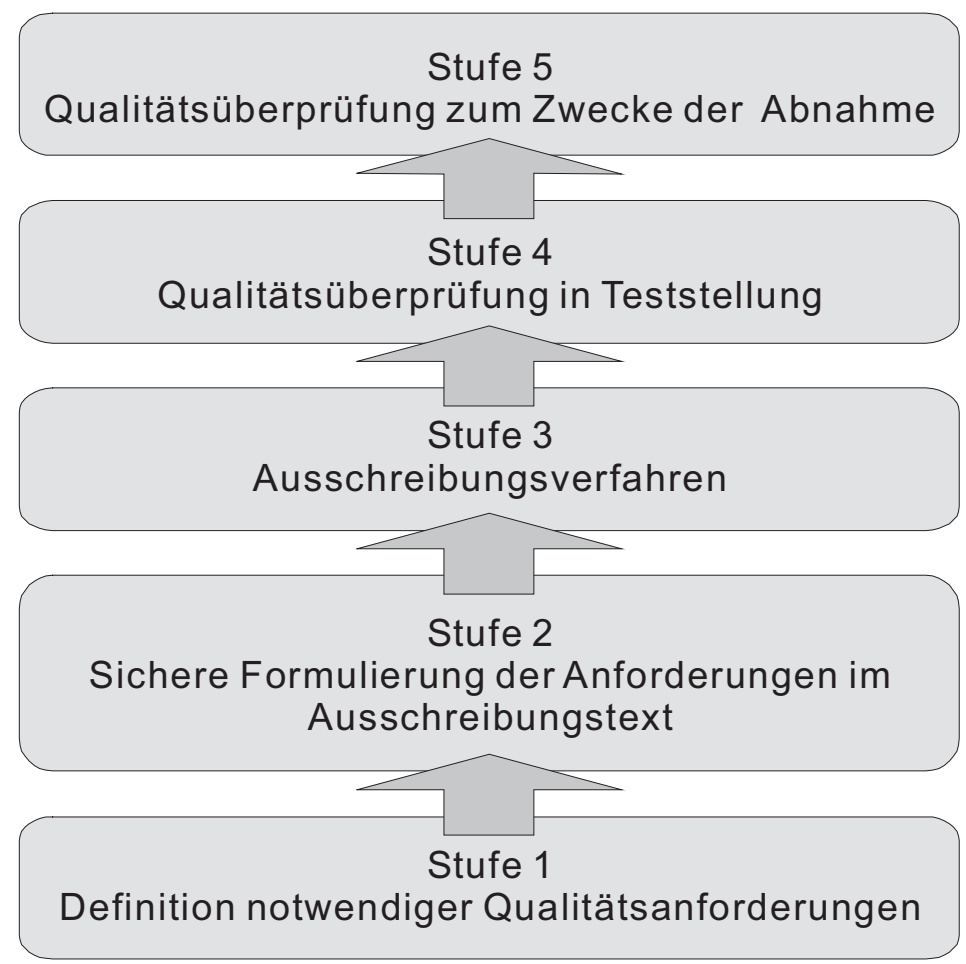

Abb. 3: Qualitätssicherungsmaßnahmen für alle Projektphasen

Um die zuvor genannten Ziele zu erreichen, wird in der ersten Stufe eine Anforderungsanalyse vorgenommen. Hier wird genau ermittelt, welche Eigenschaften bei welchem Gerät den Kann-, Soll- bzw. Muss-Anforderungen zu gerechnet werden. In der zweiten Stufe müssen die ermittelten Eigenschaften präzise und rechtssicher für die Ausschreibung festgeschrieben werden. 
In der dritten Stufe ist eine Teststellung des jeweiligen Systems sinnvoll. Es erfolgt eine erste Funktions- und Leistungsüberprüfung, bei der analysiert wird, ob sich die Anforderungen der Ausschreibung (Soll-Werte) mit den Kennzahlen des Testsystems (Ist-Werte) decken und ob Eigenschaften wie in der Dokumentation zugesichert eingehalten werden. Durch vertiefende Untersuchungen des jeweiligen Systems können die Anforderungen der Ausschreibung an dieser Stelle dann noch weiter präzisiert werden.

In der vierten Stufe erfolgt dann die Auftragserteilung mit der zeitlich nachgelagerten Anlieferung der Systeme. Bei der Anlieferung unterlaufen die Geräte dann eine einfache Funktionsüberprüfung. Hier soll sichergestellt werden, dass sich die Geräte in einem, für den späteren Betrieb einwandfreien Zustand befinden. Die Prüfverfahren und Tests, die dabei zum Einsatz kommen, sind an die Praxis angelehnt.

In der fünften Stufe, der Abnahme, sollten die angelieferten Teilsysteme eindeutig identifiziert und auf besonders wichtige Merkmale und Eigenschaften hin im Detail untersucht werden. Abnahme- und Produktionsumgebung sind dabei identisch. Definierte und abgestimmte Abnahmekriterien stellen sicher, dass die vertraglichen Forderungen hinsichtlich Leistungserbringung und Funktionserfüllung im Sinne der Bibliothek bei der Abnahme von Systemteilen erfüllt werden, damit Mängel durch rechtzeitige Feststellung und Analyse von Fehlern verhindert werden können. Werden alle Anforderungen der Bibliothek hinsichtlich der realisierten Merkmale des Produktes erfüllt, gilt die Abnahme als bestanden.

Als Abgrenzung gegenüber den Prüfverfahren zur Feststellung, ob ein Prüfgegenstand eine oder mehrere vereinbarte, vorgeschriebene oder erwartete Bedingungen erfüllt, werden Tests benötigt. Diese zeichnen sich dadurch aus, dass bei ihnen in einem standardisierten Verfahren unter bestimmten definierten Bedingungen individuelle Funktions- und Spezifikationsmerkmale gemessen werden, um später Schlüsse auf die Eigenschaften und auf das Verhalten von Systemen in anderen (ähnlichen) Situationen ziehen zu können.

Der Vorgang des Messens an sich hat dabei in beiden Verfahren einen experimentellen Charakter bei der nach DIN 1319 ein spezieller Wert einer physikalischen Größe als Vielfaches einer Einheit oder eines Bezugswertes ermittelt wird.

Tests als standardisierte Verfahren haben darüber hinaus den Vorteil, dass im Fall von Meinungsunterschieden zum Erbringungsstand von Leistungen und bei Problemen bei der Abnahme, die Rechtsprechung sich erfahrungsgemäß am Stand der Technik orientiert, das heißt, in der Regel an den dazu vorliegenden Normen und Richtlinien. Dies ist ein wesentlicher Grund, weshalb die TH Wildau die Bemühungen vorantreibt, diesen Stand der Technik von RFID-Bibliothekssystemen in Form einer VDI Richtlinie (4478) zu beschreiben. Diese sollen dann mit Beurteilungs- und Bewertungskriterien fundierte Entscheidungshilfen liefern und somit den Maßstab für einwandfreies technisches Vorgehen bei der Qualitätssicherung von RFID-Systemen definieren.

Ausgehend von seiner Komplexität ist ein RFID-System als IT-System anzusehen, da es sich um ein dynamisches und technisches System mit der Fähigkeit zur Speicherung und 
Verarbeitung von Informationen handelt. Es umfasst somit nicht nur die RFID-Hardware, sondern auch alle Komponenten der Hard- und Software sowie des Netzwerkes, die für die Kommunikation mit dem Library Management System (LMS) notwendig sind.

Für IT-Systeme beschreibt ISO 90003 in verschiedenen Abschnitten phasenunabhängige Aktivitäten. Besonders der Abschnitt der Integration/Systemtests bzw. Betrieb/Wartung kann hier auf die Aktivitäten der RFID-Einführung in Bibliotheken übertragen werden. Dabei kommen folgende Prüfmethoden in Betracht:

- Blackbox-Text

- Whitebox- Test

- Systemtest

- Stresstest/Lasttest

- Integrationstest

- Installationstest

- Abnahmetest

- Feldtest

Ein Blackbox-Test ist ein Testverfahren bei dem Tests ohne Kenntnisse der Implementierung vorgenommen werden. Beim gegenteiligen Whitebox-Test werden Kenntnisse über die Funktionsweise des Systems vorausgesetzt. In Bezug auf RFID-Systeme in Bibliotheken erfolgen die meisten Tests nach der Blackbox-Methode, da die inneren Strukturen und Abläufe der Systeme unbekannt sind. Lediglich die Funktions- und Wirkweise der jeweiligen RFID-Komponente kann als bekannt vorausgesetzt werden. Unter Systemtest versteht man einen Test des fertigen Systems gegen die in den Anforderungsdokumenten festgelegten Funktions-, Leistungs- und Qualitätsanforderungen. Dies setzt eine konventionelle, funktionsorientierte Testplanung voraus, in der die Testfälle auf Grundlage der Anforderungen an die RFID-Systeme entwickelt werden. Dem Systemtest unterzuordnen sind die Stress- bzw. Leistungstests, in denen Systeme hinsichtlich ihrer Funktionalität in Grenzbereichen untersucht werden bzw. deren Leistungsverhalten überprüft wird. Hier werden dieselben Anforderungen überprüft, jedoch befindet sich das System in einer Lastsituation bzw. wurden ihm Ressourcen entzogen.

Der Begriff Integrationstest bezeichnet eine aufeinander abgestimmte Reihe von Einzeltests, die dazu dienen, verschiedene voneinander abhängige Komponenten eines komplexen Systems im Zusammenspiel zu testen. Beim Installationstest wird geprüft, ob das System mit den Installationsbeschreibungen, die beispielsweise im Benutzerhandbuch dokumentiert sind, installiert und in Betrieb genommen werden kann. Ist dies erfolgreich, erfolgt meist anschließend der Abnahmetest, bei dem das System gegen die Produktdefinition getestet wird. Auftretende Fehler werden dabei in einem Abnahmeprotokoll dokumentiert. Wenn die Fehler tolerierbar sind oder korrigiert werden können, dann erfolgt die Abnahme des Systems durch den Auftraggeber. Andernfalls wird die Abnahme verschoben und das System muss nachgebessert werden.

Bei einem Feldtest handelt es sich um ein Verfahren, das die Qualität eines Produktes unter nicht manipulierbaren Realbedingungen testet. Dies ist dann der Fall, wenn das System vor Ort in der Bibliothek installiert wurde. 
Zur Sicherstellung der Qualität solcher IT-Systeme bedarf es eines Qualitätsmanagements mit einer den Aufgaben entsprechend angepassten Aufbauorganisation, die für die einzelnen Projektstadien die richtigen Kompetenzen eindeutig zuordnet. Idealerweise werden die dafür beauftragten Personen der Projektleitung unterstellt und besitzen je nach Projektabschnitt leitende, ausführende, bzw. prüfende Funktion.

\section{Prüfvorschriften und Richtlinien und Standards}

\section{Stand der Entwicklung}

Unterstützt wird diese Entwicklung durch mehrere Standardisierungsinitiativen, die sich im Bereich RFID mit Standardisierungen und Normung in den Bereichen Technologie, Kommunikation, Daten und Anwendungen auseinander setzen. Standards sind dabei technisch-ökonomische Vorschriften, in denen einheitlich anzuwendende Lösungen festgelegt sind. Sie regeln, wie Systeme in Bezug auf die Qualität und zu verwendende Rohstoffe und Materialien beschaffen sein müssen, bzw. welche technischen und ökonomischen Forderungen sie erfüllen müssen; sie haben empfehlenden Charakter. Ein Standard, der auf nationaler oder internationaler Ebene durch ein Normungsverfahren als allgemein gültig und rechtlich anerkannt und veröffentlicht wird, bezeichnet man als Norm. Sie besitzt die größte Verbindlichkeit einer aufgeführten Spezifikation. Eine Norm ist eine anerkannte Regel, die insbesondere in der Technik zu einer Vereinheitlichung von Eigenschaften, Produkten oder Prozessen führen soll und dabei bestimmenden Charakter besitzt.

Daneben existieren noch weitere richtungsweisende, praktische Arbeitsunterlagen vom Verein Deutscher Ingenieure e.V. (VDI), die den Stand der Technik widerspiegeln (siehe Tabelle 1). Diese VDI-Richtlinien vereinheitlichen die Anforderungen an materielle und immaterielle Güter, schaffen Vergleichbarkeit und vermeiden Anpassungskosten. Im Bezug auf Abnahmerichtlinien bezüglich Schleusensysteme gibt es die VDI 4470 in deren Anlehnung auch die Prüfverfahren an RFID-Gates entwickelt wurden.

\begin{tabular}{|l|l|}
\hline \multicolumn{2}{|l|}{ Technische Standards } \\
\hline $\begin{array}{l}\text { DIN EN 62369-1 / } \\
\text { VDE 0848-369-1 }\end{array}$ & $\begin{array}{l}\text { Ermittlung der Exposition von Personen gegenüber elektromagnetischen } \\
\text { Feldern im Frequenzbereich 0 Hz bis 300 GHz durch Geräte mit kurzer } \\
\text { Reichweite }\end{array}$ \\
\hline $\begin{array}{l}\text { DIN EN 50364 } \\
\text { VDE 0848-364 }\end{array}$ & $\begin{array}{l}\text { Begrenzung der Exposition von Personen gegenüber elektromagnetischen } \\
\text { Feldern von Geräten, die im Frequenzbereich von 0 Hz bis 300 GHz } \\
\text { betrieben werden }\end{array}$ \\
\hline ISO/IEC 15691 & $\begin{array}{l}\text { RFID for Item Management Datenformat und Speicherung } \\
\text { Data encoding Rules and logical Memory functions }\end{array}$ \\
\hline ISO/IEC 15692 & RFID for Item Management - Datenprotokoll Anwendungsschnittstelle \\
\hline ISO/IEC 15693 & $\begin{array}{l}\text { Regelung für eindeutige Ident-Nummern (»Unique Identifier«) } \\
\text { für Transponder beziehungsweise Tags }\end{array}$ \\
\hline ISO/IEC 18000-1 & Luftschnittstelle - Referenzarchitektur und Parameterbeschreibung \\
\hline ISO/IEC 18000-3 & Luftschnittstelle 13.56 MHz \\
\hline ISO/IEC TR 24710:2005 & $\begin{array}{l}\text { Elementary tag license plate functionality for ISO/IEC 18000 air interface } \\
\text { definitions - Elementartransponder }\end{array}$ \\
\hline
\end{tabular}




\begin{tabular}{|c|c|}
\hline \multicolumn{2}{|l|}{ Technische Standards } \\
\hline $\begin{array}{l}\text { ISO/IEC 18046:2006 } \\
1 / 2 / 3\end{array}$ & Leistungstests von RFID-Systemen \\
\hline ISO/IEC 18047-3:2004 & Konformität der Luft-Schnittstelle 13,56 MHz \\
\hline ETSI EN 300330 & Funkparameter im Bereich $9 \mathrm{kHz}$ bis $30 \mathrm{MHz}$ \\
\hline ETSI EN 301489 & Elektromagnetische Kompatibilität \\
\hline VDI $4470 / 1$ & $\begin{array}{l}\text { Warensicherungssysteme - Kundenabnahmerichtlinie für Schleusensysteme } \\
\text { Ermittlung der Erkennungsrate und Detektionsrate bei der Inbetriebnahme } \\
\text { von EAS-Systemen vor Ort }\end{array}$ \\
\hline VDI $4470 / 2$ & $\begin{array}{l}\text { Warensicherungssysteme - Kundenabnahmerichtlinie für Deaktivierungs- } \\
\text { anlagen« Prüfung von Deaktivierungsanlagen für EAS-Systeme Abnahme } \\
\text { mit realen Produkten }\end{array}$ \\
\hline VDI $4470 / 3,4$ & $\begin{array}{l}\text { Warensicherungssysteme - Kundenabnahmerichtlinie für Deaktivierungs- } \\
\text { anlagen Prüfung von Deaktivierungsanlagen für EAS-Systeme Abnahme mit } \\
\text { künstlichen Produkten }\end{array}$ \\
\hline VDI 4472/2 & $\begin{array}{l}\text { Anforderungen an Transpondersysteme zum Einsatz in der Supply Chain - } \\
\text { Allgemeiner Teil }\end{array}$ \\
\hline VDI/AIM 4472/10 & $\begin{array}{l}\text { Testverfahren zur Überprüfung der Leistungsfähigkeit von Transponder- } \\
\text { systemen (RFID) }\end{array}$ \\
\hline VDI 4471 & Anforderungen an Transpondersysteme zum Einsatz in der Supply Chain \\
\hline VDI 4478 & Anforderungen an RFID-Systeme für den Einsatz in Bibliotheken (in Arbeit) \\
\hline VDI 4478-1 & $\begin{array}{l}\text { Testverfahren zur Vereinheitlichung der Leistungsbestimmung von RFID- } \\
\text { Gates für den Einsatz in Bibliotheken (erschienen 2012) }\end{array}$ \\
\hline VDI 4478-2 & Leistungsbestimmung von RFID-Label für den Einsatz in Bibliotheken (in Arbeit) \\
\hline \multicolumn{2}{|l|}{ Qualitätsstandards } \\
\hline DIN 1319 & Deutsche Norm in der Messtechnik - Grundbegriffe, \\
\hline DIN EN ISO 9000 & Grundlagen von Qualitätsmanagementsystemen \\
\hline DIN EN ISO 9001 & Anforderungen an ein Qualitätsmanagementsystem \\
\hline DIN EN ISO 9004 & Effizienz von Qualitätsmanagementsystemen \\
\hline VDI 26191985 & Prüfplanung Arbeitsschritte zur Erstellung eines Prüfplanes \\
\hline DIN 32937 & Mess- und Prüfmittelüberwachung \\
\hline ISO /IEC 90003:2004 & Software Engineering ISO 9000 für Software \\
\hline
\end{tabular}

Tab. 1: Standards/Normen/Richtlinien im Bereich RFID/Qualitätsmanagement

\section{VDI Richtlinienarbeit 4478}

Mit der Einrichtung eines Runden Tisches an der TH Wildau zur Erarbeitung von Prüfvorschriften zur Messung der Leistungsfähigkeit von RFID Gates Systemen (13,56 MHz) ist der Startschuss für die Erstellung offizieller VDI Richtlinien gefallen. Mit der Richtlinienrahmennummer 4478 können nun die notwendigen Beschreibungen des Stands der Technik erfolgen. Begonnen wurde mit der Betrachtung der Gatesysteme, die hinsichtlich der Funktionalität die größte technische Herausforderung darstellen.

Naturgemäß entwickeln sich Richtlinienwerke weiter und es werden andere systemrelevante Fragestellungen behandelt. So ist in der Abb. 4 ein möglicher Aufbau des Richtlinienwerkes VDI 4478 RFID Einsatz in Bibliotheken skizziert.

Für die internationale Nutzbarkeit der VDI Richtlinie ist es von Bedeutung, dass der Richtlinienausschuss international besetzt ist und eine deutsch-englische Ausgabe von Beginn zur Verfügung steht. Des Weiteren ist der Verband der Industrie der 
Automatischen Identifikation und Mobilen Datenerfassung AIM e.V. bzw. dessen Mutterorganisation AIM Global mit Sitz in den USA, autorisiert, direkte Eingaben beim ISO vorzunehmen. Es wird angestrebt, den AIM für Einbringung der VDI Richtlinie in den internationalen Standardisierungsprozess zu gewinnen.

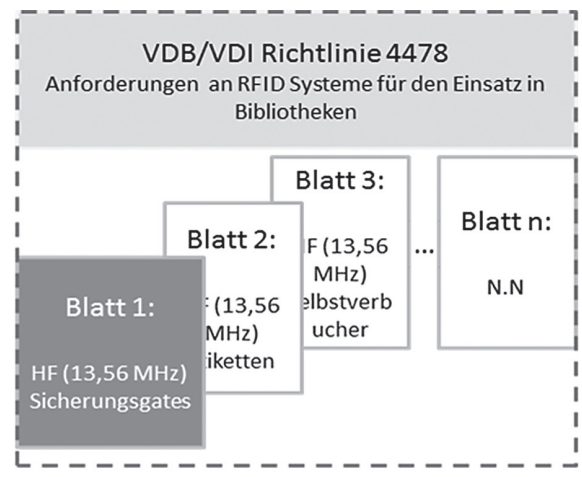

Testverfahren zur Vereinheitlichung der Leistungsbestimmung von RFIDKomponenten für den Einsatz in Bibliotheken (Benchmarktest)

HF (13,56 MHz) Sicherungsgates

Abb. 4: Möglicher Aufbau des Richtlinienwerkes 4478

Der Nutzen derartiger Richtlinien und Standards ist eindeutig in einer Professionalisierung der Anwendung von RFID in Bibliotheken zu sehen, d. h. in der Perfektionierung des Marktes hin zu einem transparenten Markt.

Im Einzelnen können folgende Aspekte des Nutzens nach Interessensgruppen genannt werden:

\section{Nutzen für die Anwender}

Die Vorgehensweise bietet für die Anwender vielfältigen und nachhaltigen Nutzen.

- Unterstützung bei der Definition wichtiger qualitätsbestimmender Eigenschaften

- Vereinfachung von Ausschreibungen durch Bezug auf Richtlinien und Normen oder auch die Forderung von Nachweisen einer bestimmten Qualität z. B. in Form von Zertifikaten und Prüfprotokollen

- Vergleichbare Leistungsmetrik zur Unterstützung von Ausschreibungsentwürfen

- Nachweisbare Berücksichtigung des Stands der Technik, d. h. verbesserte Prüfungsfestigkeit in den Vergabeverfahren

- Einheitliche Terminologie zur verbesserten Kommunikation mit den Anbietern

- Reduzierte Einarbeitung von Personen in detaillierte technische Vorgänge

- Verlässliche Abnahmeprozeduren vor Ort

- Reproduzierbare Abläufe während der Abnahme und des täglichen Betriebs

- Schnellere Diagnose von Ursachen bei Ausfällen/Leistungsminderungen 


\section{Nutzen für die Anbieter}

Die Vorgehensweise bietet für die Anbieter ebenfalls vielfältigen und nachhaltigen Nutzen.

- Vereinfachte Kommunikation von Differenzierungsmerkmalen der Produkte

- Anforderungsoptimierte Angebote für robuste Preispolitik

- Beschleunigte Vorortabnahmen führen zu geringeren Implementierungskosten

- Standardisierte Schulungen des technischen Personals der Anwender

- Tragfähige Schulungen können von Dritten angeboten werden

- Standardisierbare Diagnose-Modelle führen zu einer Optimierung des Serviceangebotes (1st, 2nd, usw. Level Support)

- Offizielle zweisprachige Richtlinien des VDI führen zur Beeinflussung auch des europäischen Marktes und führen zu Synergien

- Adaption durch ISO

- Vereinfachte Positionierung am Markt

\section{Begriffsbestimmung}

Um Medien wirkungsvoll vor Diebstahl zu schützen, werden RFID-Sicherungsgates im Zugangsbereich zur Bibliothek bzw. zu den Lesesälen installiert. Über eine Kennzeichnungsmöglichkeit auf den Transpondern können diese unterscheidbar gruppiert werden. Die Alarmgenerierung über diese Merkmale erfolgt in Deutschland meist durch Programmierung des AFI (application family identifier) auf dem Transponder. Im Zuge der Mediensicherung sendet ein Gate einen entsprechenden Abfragebefehl, auf den dann die mit dem entsprechenden AFI-Wert programmierten Transponder reagieren. Dies stellt nur die Alarmfunktionalität und die Abfrage der eindeutigen Transpondernummer (UID) sicher. Weitere Informationen zum Medium selbst müssen zusätzlich vom Transponder oder aus einer Datenbank ausgelesen werden. In anderen Regionen wird oft die Erkennung mittels EAS-Verfahren eingesetzt. Dabei reagiert jeder Transponder im Gate, bei dem die EAS-Alarmfunktion frei geschaltet wurde. Dieses Verfahren bietet zwar den Vorteil, dass die Alarmierung sicher ausgeführt wird, hat aber gleichzeitig den Nachteil, dass die UID oder andere Mediendaten nicht ermittelt werden. Es ist somit nicht unterscheidbar, ob der alarmauslösende Transponder aus der betreffenden Bibliothek stammt und auch nicht, um welche Art Medium es sich handelt. Nach der Alarmauslösung müssen also andere Prozesse folgen, um eine höhere Sicherheitsstufe zu erreichen.

\section{Analyse der funktionsbestimmenden Parameter}

Die Erkennungsrate ist das Hauptmerkmal bei der Beurteilung von RFID-Schleusensystemen. Die Erkennungsrate stellt sich dabei als Verhältnis von erfassten zu erfassbaren Transpondern dar. Sie sollte im Idealfall hundert Prozent betragen, nämlich dann, wenn immer alle Transponder detektiert werden. Dies ist jedoch in der Realität nicht immer der Fall. Aus theoretischer Sicht gibt es mehrere Faktoren, die zu einer Beeinflussung der Detektion beitragen und sich somit auf die Erkennungsrate auswirken: 
Zum einen ist die Verweildauer der Transponder innerhalb des Erfassungsbereiches des Gates zu nennen; da die Transponder durch spezielle Zugriffsverfahren sequentiell gelesen werden, muss diese ausreichend hoch sein. Die notwendige Verweildauer wird wiederum beeinflusst durch die Anzahl der Transponder. Geht man davon aus, dass für die Detektion eines Transponders eine bestimmte Zeit benötigt wird, so summieren sich die Zeiten, wenn mehrere Transponder verwendet werden. Sollen zusätzlich zur AFIErkennung noch weitere Daten vom Transponder gelesen werden, muss die Verweildauer für das Auslesen angepasst werden.

Die Verweildauer wird durch die Geschwindigkeit bestimmt, mit der die Transponder durch den Erfassungsbereich bewegt werden. Die Verweildauer ist hier umso kürzer, je schneller die Transponder durch das Gate bewegt werden. Die Strecke, die dabei innerhalb des Erfassungsbereiches zurückgelegt wird, ist abhängig von Antennengröße bzw. von der Gate-Tiefe. Diese sind bei einem installierten Gate konstante Produktmerkmale.

Ein weiterer Faktor, der Einfluss auf die Detektion und damit Erkennungsrate hat, ist die Homogenität und die Ausbreitung des magnetischen Feldes. Eine optimale Erfassung von Transpondern kann nur dann erreicht werden, wenn zwischen zwei Gate-Antennen eine lückenlose Ausbreitung des Feldes erreicht wird, sodass an jeder Position genügend Energie bereitsteht, um Transponder mit Energie zu versorgen und eine Kommunikation aufzubauen. Auch spielt die Lage der Transponder innerhalb des magnetischen Feldes eine Rolle; je nach Bauform der Gate-Antenne baut sich um die Antennenwindungen ein charakteristisches Feld auf. Die Koppeleigenschaften von magnetisch gekoppelten Systemen hängen jedoch maßgeblich von der mechanischen Anordnung zueinander ab. Eine Neigung des Transponders gegenüber den Feldlinien vermindert die Durchdringung der Transponderspule durch die Feldlinien des Gates und es kann im ungünstigsten Fall keine Energieversorgung beziehungsweise keine Kommunikation mehr stattfinden. Deshalb ist es wichtig, Prüfverfahren zu entwickeln, welche die Lage von Transpondern berücksichtigen. Damit der Aufwand noch akzeptabel ist, werden für einen Transponder die 12 wichtigsten möglichen Lagen definiert (Abb. 4). Ausgehend von einem dreidimensionalen kartesischen Koordinatensystem sind dies 6 Hauptlagen und weitere 6 Hauptlagen, die jeweils um $45^{\circ}$ gedreht wurden.
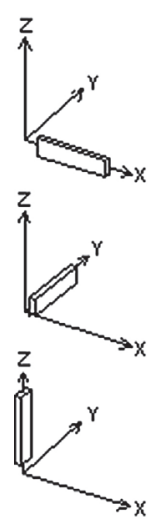
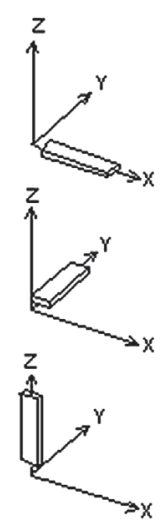
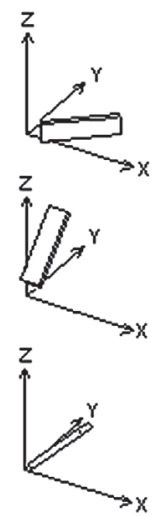
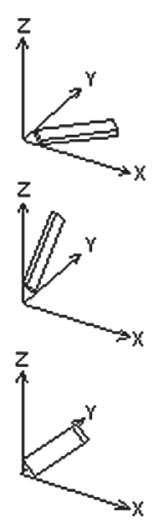

Abb. 4: Darstellung der 12 Transponderlagen [VDI4470] 
Die Homogenität des Feldes kann beeinträchtigt werden, wenn der Abstand der GateAntennen so groß ist, dass eine vollständige Durchflutung des Durchgangsbereiches nicht mehr erreicht werden kann. Die Reichweite der Antennen von Gates ist deshalb ein prüfenswerter Parameter.

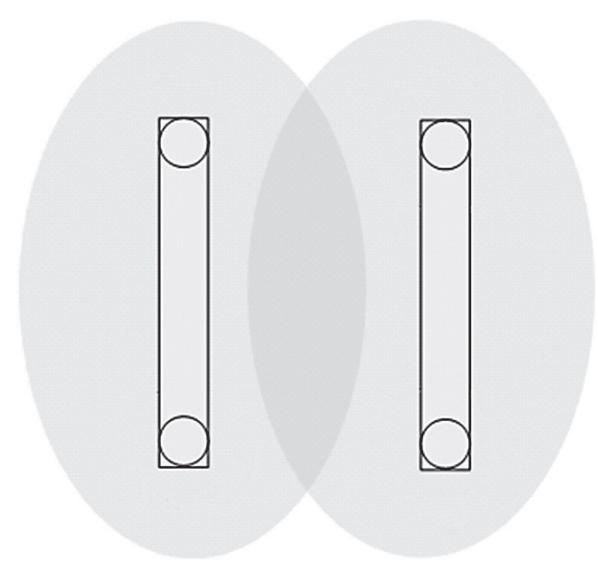

Abb. 5: Feldhomogenität bei einem RFID-Gate (Draufsicht)

Eine optimaler Antennenabstand, wie vom Hersteller empfohlen, kann in den Bibliotheken aufgrund von baulichen und architektonischen Restriktionen sowie Vorschriften nicht immer realisiert werden. Typische Durchgangsbreiten von Gates innerhalb der Bibliotheken bewegen sich daher in Bereichen zwischen 90 und $120 \mathrm{~cm}$. Bei großen Abständen als vom Hersteller des Gates vorgegeben können jedoch Lücken im Detektionsbereich auftreten, in denen eine Detektion von Transpondern nicht mehr möglich ist. Prüfverfahren, bezüglich der Durchgangsbreite müssen daher die gegebenen Antennenabstände vor Ort berücksichtigen.

Die Hauptfunktion eines RFID-Sicherungsgates, die Mediensicherung, sollte auch im Sinne des Diebstahlschutzes getestet werden. Dabei sollte die Diebstahlsituation so geprüft werden, wie sie auch in der Realität ablaufen würde. Die Auswahl der Medien und der Transportverstecke sollte typischerweise heterogen erfolgen und sich an bereits bekannten Diebstählen aus dem Bibliotheksalltag orientieren. Diebstahlstatistiken, sofern erfasst, könnten dabei wichtige Kennzahlen liefern.

Eine optional vorhandene Besucherzählung muss hinsichtlich der Erkennungsrate untersucht werden, da sie die Genauigkeit der Zählfunktion widerspiegelt.

Zusammenfassend ergeben sich für das Teilsystem RFID-Gate folgende qualitätsbestimmende Parameter:

- Feldhomogenität

- Pulkfähigkeit

- Erkennungsrate in Abhängigkeit der Geschwindigkeit

- Erkennungsrate in Abhängigkeit der Transponderlage

- Diebstahlschutz

- Erkennungsrate / Besucherzählung 


\section{Umsetzung der Prüfverfahren}

\section{Hintergründe}

Nach der Analyse der wesentlichen Parameter ist es notwendig Prüfverfahren zu entwickeln, die folgende Eigenschaften besitzen:

- Lieferung von wiederholbaren Ergebnissen unter gleichen Umweltbedingungen (Reproduzierbarkeit)

- Nachvollziehbarkeit der Ergebnisse und Zusammenhang mit der Praxis

- Generierung eines breiten wahrnehmbaren Spektrums der Ergebnisse von unterschiedlichen Prüflingen

- Trennung der zu untersuchenden Faktoren

- kurze Testzeit

- geringer Testaufwand

- Akzeptanz durch die relevanten Marktteilnehmer

- große Präzision als Labortest

- gute Ableitbarkeit von Tests für die reale Umgebung vor Ort

Diese Eigenschaften oder besser Anforderungen an die Testprozeduren lassen sich in der Realität nicht vollständig erfüllen. Ein Beispiel: Theoretisch ist zwar eine gute Trennung der zu testenden Eigenschaften möglich, das würde aber zu einer Vielzahl von Einzeltests führen, was den Aufwand schnell in unrentable Höhen geraten lässt. Hinzu kommt noch, dass eine detaillierte Austestung von abgegrenzten Eigenschaften zwar für Hersteller oder Entwickler interessant, aber die praktische Relevanz für die Schaffung einer Entscheidungs- und Diagnosehilfe fragwürdig ist.

Zum optimalen Erreichen der o. g. Eigenschaften der Prüfverfahren ist es notwendig, Expertenwissen aus einer repräsentativen Anzahl von Marktteilnehmern zu bündeln. Dafür wurde, wie oben bereits erwähnt, an der TH-Wildau der Runde Tisch Bibliothekssysteme gebildet. Gleichzeitig wurde aktiv Kontakt zur Gruppe der Anwender von RFIDBibliothekssystemen aufgenommen.

Über einen hinreichenden Zeitraum wurde dann ein Optimum an Tests für RFID-Sicherungsgates erarbeitet. Die in der VDI-Richtlinie VDI 4478-1 festgeschriebenen Verfahren stellen eine Gesamtheit dar. Sie bieten einen optimalen Überblick über die Qualität der getesteten Gates, sind von einer repräsentativen Menge von Marktteilnehmern akzeptiert, liefern gut gefächerte Ergebnisse und sind aufwandoptimiert.

Für diese Optimierung war es erforderlich, Abstriche an die Trennung der einzelnen qualitätsbestimmenden Merkmale zu machen. Es sind die folgenden Einzeltests definiert:

- Bestimmung von elektrischen Feldparametern zur Festschreibung des Zustandes des Prüflings während des Tests

- Bestimmung der Homogenität des Erfassungsfeldes über die Fläche des Durchgangsbereiches

- Bestimmung der Detektionszuverlässigkeit mit 18 Transpondern bei verschiedenen Geschwindigkeiten 
- Bestimmung der Möglichkeit Transponder von Medienstapeln zu detektieren

- Untersuchung der Stabilität der Erkennungsrate unter dem Einfluss von aktiven und passiven Störern

Diese Einzeltests sind für die Detektionsverfahren mittels AFI und EAS definiert und entsprechend angepasst. Bei der Optimierung der Einzeltests wurde der Reproduzierbarkeit eine sehr hohe Priorität eingeräumt. Diesem Kriterium und auch der Praxisnähe sind einige anfangs vorgeschlagene Tests zum Opfer gefallen.

Eingangs wurden in der Richtlinie die einzusetzenden Prüfkörper und das nötige Testumfeld beschrieben.

Um die definierten Prüfungen effizient und wiederholgenau durchführen zu können, wurde an der TH-Wildau eine Bewegungsapparatur entwickelt und hergestellt. Sie ist in der Lage verschiedene Prüfkörper wiederholgenau mit hinreichender Präzision in Geschwindigkeit, Bahn und Lage durch das zu prüfende Gate zu verfahren. Die Bewegungen werden dabei ferngesteuert vom Prüfarbeitsplatz veranlasst.

Die Abmessungen der Apparatur sind mit $4 \mathrm{~m}$ x $3 \mathrm{~m}$ x 2,5 m deutlich größer als die des Prüflings selbst. Es ist schließlich sicher zu stellen, dass die Prüfkörper unter Beachtung des Beschleunigungs- und Bremsweges innerhalb des Erfassungsbereiches des Gates die vorgegebene Geschwindigkeit besitzen.

Der Aufbau der Apparatur erfolgte aus Nichtmetallen. Dadurch ist sichergestellt, dass die Eigenschaften des Prüflings während der Prüfung nicht durch das Hilfsmittel verändert werden.

Bei der elektrischen Verkabelung wurde darauf geachtet, dass der Prüfling nicht von Fremdfeldern beeinflusst wird.

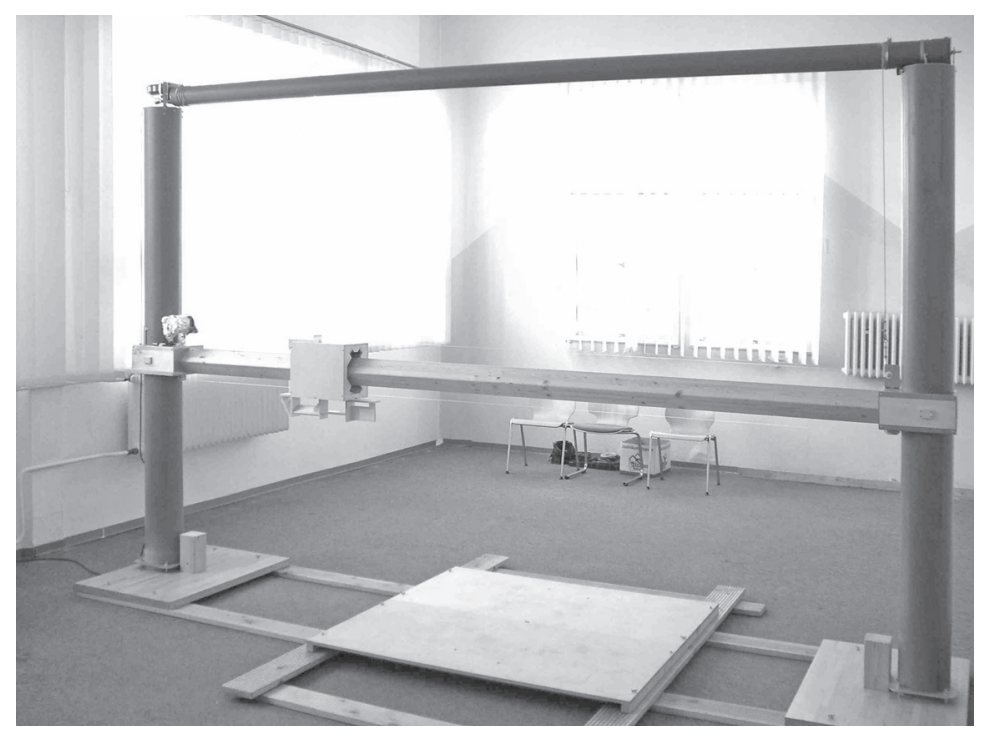

Abb. 6: Bewegungsapparatur 


\section{Homogenität des Erfassungsfeldes}

Für diesen Test wurde ein Prüfkörper definiert, der aus 5 Transpondern besteht, die gut abgestimmt sind und sich hintereinander in einer Linie in verschiedenen Ausrichtungen befinden. Dieser Prüfkörper wird auf einer größeren Anzahl genau definierter Verfahrlinien mit einer definierten Geschwindigkeit durch das Gate bewegt. Man erhält Aussagen darüber, wie gut das Erfassungsfeld innerhalb des Gates über den Durchgangsbereich verteilt ist.

\section{Detektionszuverlässigkeit}

Dieser Test wird mit einer größeren Anzahl von Transpondern (18 Stück) innerhalb des Prüfkörpers mit unterschiedlichen Geschwindigkeiten durchgeführt. Er stellt die Eigenschaften des Gates unter erhöhter Belastung dar. Durch den Einsatz von unterschiedlichen Geschwindigkeiten wird deutlich, ob sich ein Gate z. B. an seiner Leistungsgrenze befindet. Um die Reaktion des Gates unter »Belastung « auf unterschiedliche Transponderausrichtungen festzustellen, wird der Prüfkörper in drei verschiedenen Ausrichtungen durch das Gate verfahren. Die Anzahl der Verfahrlinien wurde zur Verkürzung der Testzeit reduziert.

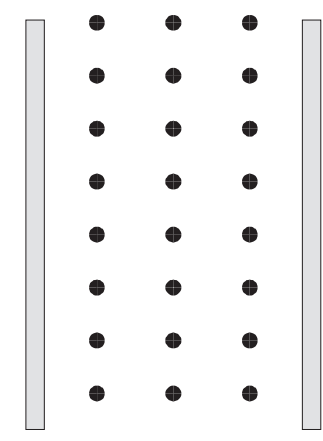

Abb. 7: Verfahrlinien

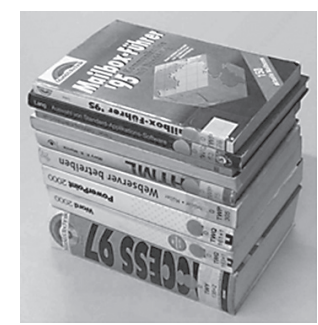

Abb. 8: Buchstapel mit Metalleinband oder ähnliche Exoten verzichtet. Anschließend wurden die maßgeblichen Eigenschaften dieses Stapels bestimmt und ein Prüfkörper definiert, der diese Eigenschaften repräsentiert und aus einfachen wieder beschaffbaren Mitteln hergestellt ist. So ist dafür Sorge getragen, dass auch diese Prüfung unabhängig von der Beschaffbarkeit bestimmter Medien zu späteren Zeitpunkten reproduzierbare Ergebnisse liefert.

\section{Störfestigkeit, aktiver Störer}

Zunächst wird die Erkennungsrate des zu prüfenden Gates bestimmt. Dann wird aus großer Entfernung ein definierter Störer (eingeschalteter RFID-Reader) mit festgeschriebener Feldstärke sukzessive dem zu prüfenden Gate angenähert. In den jeweiligen Entfernungsstufen wird die Erkennungsrate wiederholt bestimmt. Das Ergebnis des Tests ist die Entfernung des Störers, bei der das Gate noch die gleiche Erkennungsrate liefert wie bei der ersten Referenzbestimmung. 
Diese Untersuchung liefert Erkenntnisse über die Robustheit gegenüber Feldern von anderen RFID-Geräten, die u. U. in der Nähe des Gates installiert werden. Das Ergebnis ist jedoch nicht zu verwechseln mit der Ermittlung einer minimalen Entfernung für die geplante Installation eines RFID-Gerätes; hierfür sind weitere Gesichtspunkte heran zu ziehen.

\section{Störfestigkeit, passiver Störer}

Wird ein Gate sehr dicht an einem metallischen Element, z. B. an einem bauseitig vorhandenen Stahlträger oder einem metallischen Türrahmen installiert, wird ein Teil der Feldenergie, die zum Versorgen der Transponder erzeugt wird, in dem metallischen Element in Wärme umgesetzt. Sie geht also den Transpondern verloren. Gleichzeitig wirkt dieses Metall verstimmend auf die resonanten Gate-Antennen. Sie können also nicht so unbeeinflusst arbeiten wie in einer metallfreien Umgebung.

Für diesen Test wurde ein Prüfkörper aus Stahlblech definiert, der das Vorhandensein von Metall simulieren soll. Er wird in Schritten an das Gate heran gebracht, wobei wie beim zuvor beschriebenen Test jeweils die Erkennungsrate bestimmt wird. Die Entfernung, bei der die Erkennungsrate gerade noch der Referenzmessung entspricht, stellt das Ergebnis dar. [vgl. VDI 4478-1]

\section{Anhang}

\section{Beispiel möglicher Abläufe bei der Beschaffung von RFID-Sicherungsgates}

Die Beispiele beziehen sich auf die Beschaffung innerhalb von Deutschland. Hier kommen meist Gates um Einsatz, die auf Basis des Detektionsverfahrens »AFI « die Transponder der Medien detektieren. Bild A1 zeigt ein Schema mit zwei Ablaufvarianten. Nach der ersten Variante wird die Durchgangsbreite zwischen den einzelnen Antennen vom der ausschreibenden Stelle fest vorgegeben. Die Anbieter bieten entsprechend viele Antennen an, damit die Gesamtdurchgangsbreite von z. B. 12 Metern gesichert werden kann. In der Ausschreibung wird lediglich das Zertifikat nach VDI4478-1 verlangt. Nach dessen Vorlage werden die eingehenden Angebote berücksichtigt.

Im zweiten Beispiel ist frei gestellt, wie viel Antennen der Anbieter zur Abdeckung der Gesamtdurchgangsbreite von 12 m installieren möchte. Das ermöglicht den Aufwand zu reduzieren und den technischen Möglichkeiten entsprechende größere Durchgangsbreiten zwischen den Antennen zu bekommen. Die Anbieter müssen dazu ein Zertifikat für die ggf. größere Durchgangsbreite zwischen den Antennen vorlegen. Dafür ist bei ihnen eine Zertifizierung notwendig, die über die übliche Zertifizierung für $1 \mathrm{~m}$ Durchgangsbreite hinaus geht. Wenn sie entsprechende Produkte liefern können, werden sie Interesse daran haben, die entsprechenden Zertifikate vorliegen zu haben.

Der Ablauf nach Bild A2 (drittes Beispiel) beschreibt das Vorgehen für Fälle, bei denen bestimme Eigenschaften der zu beschaffenen Sicherungsgates besonders hoch einzustufen sind. In diesen Fällen ist es ratsam die Richtlinie VDI4478-1 vor der Definition der 
zu priorisierenden Werte zu beschaffen. Hierzu ist mit dem VDI Kontakt aufzunehmen. Der Gründruck (erste Veröffentlichungsstufe) ist hier bereits ausreichend. Da das Zertifikat, welches Unternehmen erhalten, die ihre Gates nach VDI4478 testen lassen, nur ausweist, dass die derzeit gültigen Schwellwerte aller Prüfungen mindestens erreicht wurden, ist es in diesem Beispiel notwendig das Messprotokoll in der Ausschreibung zu verlangen. Hieraus lassen sich die Ergebnisse der Teiluntersuchungen erkennen. Ist z. B. ausgeschrieben, dass das angebotene Gate im Test »Stapelleistung « besonders gute Ergebnisse bringen soll, kann das anhand des Messprotokolls überprüft werden.

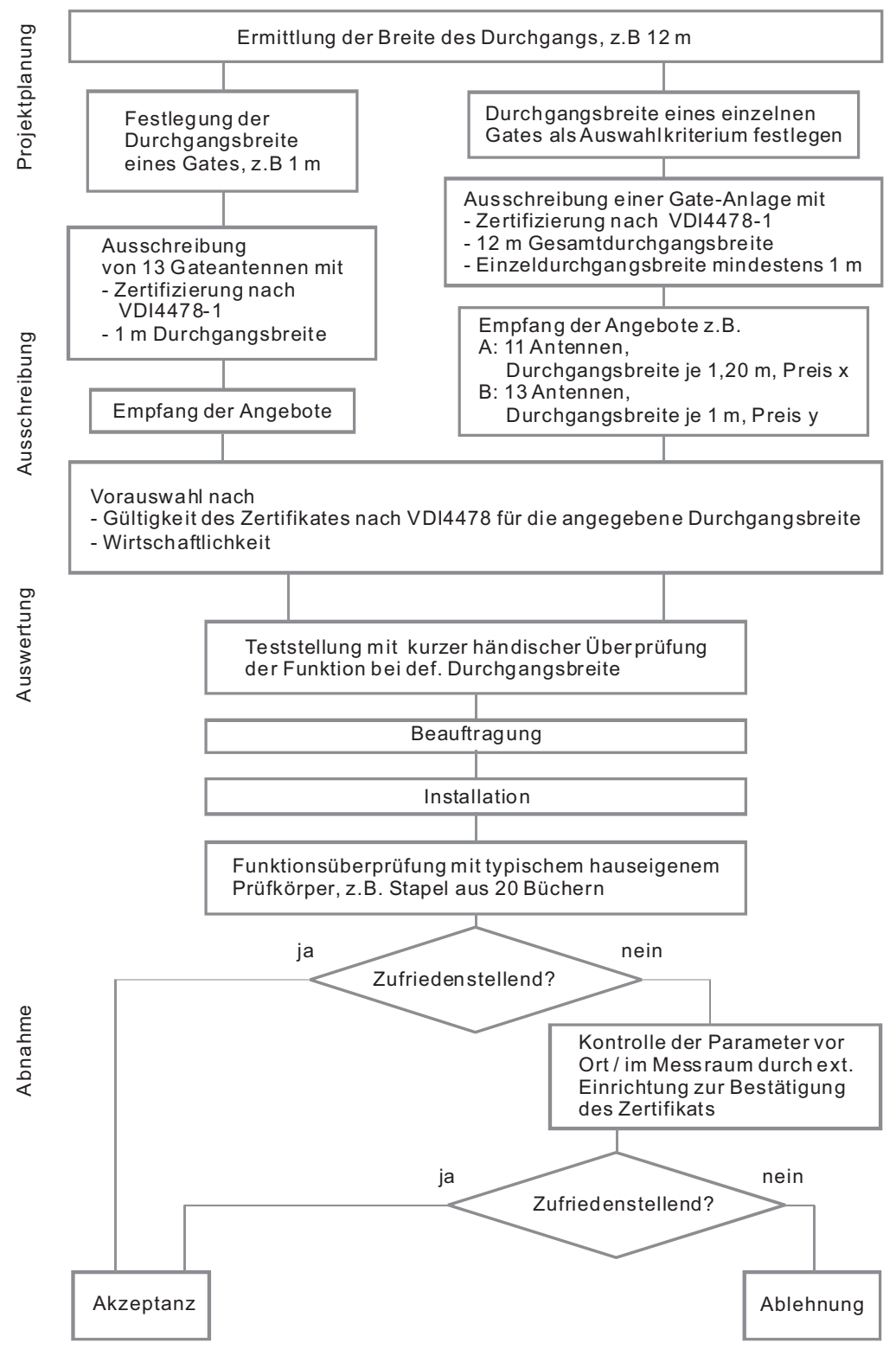

Abb. $A 1$ 


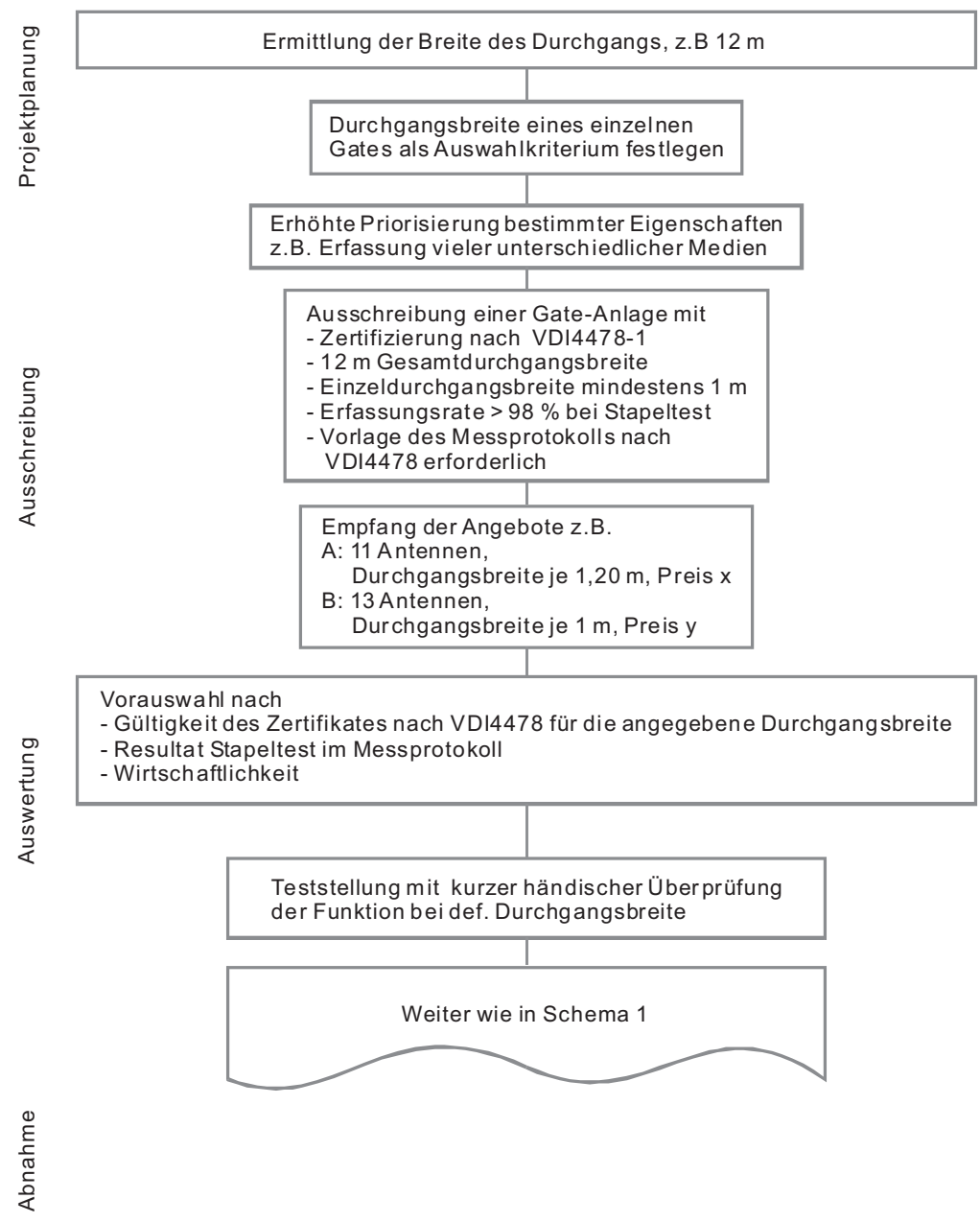

Abb. A2

\section{Quellenverzeichnis}

[Gill2007] Frank Gillert, Wolf-Rüdiger Hansen, »RFID für die Optimierung von Geschäftsprozessen«, Carl Hanser Verlag München, 2007

[ISO 8402] »Quality management and quality assurance - Vocabulary «

[VDI 4470] »Warensicherungssysteme Kundenabnahmerichtlinie für Deaktivierungsanlagen«

[VDI 4478-1] »Testverfahren zur Vereinheitlichung der Leistungsbestimmung von RFID-Gates für den Einsatz in Bibliotheken« 